\title{
Therapeutic Plasma Exchange in the Management of Myasthenia Crisis in a Resource Poor Setting
}

\author{
Akingbola T. S. \\ Department of Haematology University College Hospital (UCH), Ibadan Oyo state Nigeria, Nigeria \\ Shonde-Adebola K. B. \\ Department of Haematology University College Hospital (UCH), Ibadan Oyo state Nigeria, Nigeria \\ Anyanwu-Yeiya C. C. \\ Department of Haematology University College Hospital (UCH), Ibadan Oyo state Nigeria, Nigeria \\ Ezekekwu C. A. \\ Department of Haematology University College Hospital (UCH), Ibadan Oyo state Nigeria, Nigeria
}

Adeoye O. A.

Department of Haematology University College Hospital (UCH), Ibadan Oyo state Nigeria, Nigeria

Odebiyi H. A.

Department of Haematology University College Hospital (UCH), Ibadan Oyo state Nigeria, Nigeria

Akinyemi R.

Department of internal Medicine (Neurology Unit) UCH, Ibadan, Oyo state Nigeria, Nigeria

Kotila T. R.

Department of Haematology University College Hospital (UCH), Ibadan Oyo state Nigeriak, Nigeria

\begin{abstract}
BACKGROUND: Therapeutic Plasma Exchange (TPE) is an apheresis treatment in which the plasma component of blood is removed and replaced with supplemental fluids. It is an immunomodulatory treatment that has been reported to be a successful therapeutic procedure for the treatment of severely compromised patients with myasthenia gravis when given at short intervals. TPE is known to have a comparable efficacy to intravenous immunoglobulin (IVIG) in the treatments of patients with moderate to severe myasthenia crisis. METHODOLOGY: We report a case of a 47year old male patient with severe myasthenia crisis. He was managed using cobe spectra version 6.1 to carry out an automated TPE, with a suboptimal response and subsequent use of IVIG resulting in full recovery. This report highlights the possible factors that may hinder optimal response of a patient with Myasthenia crisis to TPE procedure in a poor resource setting. CONCLUSION: TPE is an essential immunomodulatory therapy in the management of acute myasthenia crisis whether given alone or in combination with immunosuppressive drugs. There can be a rebound overproduction of the offending autoantibodies in MG following TPE which can be cleared from circulation using immunosuppressive therapy.
\end{abstract}

Keywords: Myasthenia gravis; Myasthenia crisis; Therapeutic plasma exchange; Challenges.

(9) (1) CC BY: Creative Commons Attribution License 4.0

\section{Introduction}

Myasthenia gravis (MG) is an autoimmune disorder of the neuromuscular junction characterized by progressive fatigable weakness particularly of ocular, neck, facial and bulbar muscles. This condition is most commonly caused by autoantibodies directed against acetylcholine receptors in the post-junctional membrane of the neuromuscular junction or, much less commonly against muscle-specific tyrosine kinase (MuSK). These antibodies block neuromuscular transmission and initiate a complement-mediated inflammatory response which reduces the number of acetylcholine receptors and damages the end plate [1].

Majority of these patients present with thymic follicular hyperplasia while others have a thymoma [2]. These patients have increased incidence of other autoimmune diseases. The trigger factor for the development of the disease is unknown but some drugs have been implicated such as penicillamine, aminoglycosides and ciprofloxacin and should be avoided in patients with MG [1].

MG has annual incidence of approximately 30 new cases per million, approximately $15-20 \%$ of these patients will go into myasthenia gravis crisis (MGC) and 3-8\% of all patients who go into MGC will die [3]. Myasthenia gravis often presents between ages 15 and 50 years with a female preponderance in younger age group and a reverse in older ages [3]. Patients classically present with muscle weakness which worsens with exercise and as the day goes 
by. The first symptoms are usually intermittent ptosis or diplopia. Respiratory muscles may be involved and respiratory failure is not an uncommon cause of death. Aspiration may complicate episodes of coughing. Sudden myasthenia crisis may occur and may require ventilatory support [4]. Myasthenia crisis in patients with MG, is indicative of a generalized weakness associated with swallowing difficulties and respiratory failure often requiring intensive care treatment [5].

The principles of treatment of MG include, maximizing the activity of acetylcholine at the remaining receptors in the neuromuscular junctions (NMJ). This is achieved by inhibiting acetylcholine hydrolysing enzyme hence prolonging its action. The most common anti- acetylcholinesterase drug is pyridostigmine. Secondly, is to suppress the immunological attack on motor end plates. This requires use of immunosuppression or immunomodulation therapy such as TPE [1].

Therapeutic Plasma Exchange (TPE) is an apheresis treatment in which the plasma component of blood is removed and replaced with supplemental fluids. It is generally performed to remove substances such as autoantibodies, immune complexes, cryoglobulins and toxins that have accumulated in the plasma [6]. Its efficiency in MG is due to removal of auto-immune antibodies to acetylcholine receptor, leading to improvement of neuromuscular junction transmission, muscular strength and motor performance. The supplemental or replacement fluids used for TPE include serum albumin, Normal saline, fresh frozen plasma (FFP) etc [7]. Therapeutic plasma exchange has been reported to result in good clinical outcome in severely compromised myasthenic patients when administered in short interval (preferably on alternate day). The possible side effects of TPE include allergic transfusion reaction, features of citrate toxicity (like hypocalcemia, hypomagnesemia, muscle spasm or tetany), hypocoagulability etc. TPE has been shown to be of almost the same efficacy with IVIG as shown in a comparative study [8].

TPE is a first line treatment for MG, according to the American Society for Apheresis (ASFA) guideline for TPE. However, in spite of this fact, TPE is not readily carried out on patients with MG and other related disorders, due to limited availability of the apheresis machine in most health facilities as well as lack of trained personnel and the relative high cost of the procedure in Nigeria. In the past, before the automated apheresis became available in Nigeria, there have been reports of treatment of cases of MG crisis and Waldenstrom's macroglobulinaemia with "modified plasmapheresis" in our facility with good result. This method was though inexpensive but more laborious compared with the automated method using the apheresis machine $[9,10]$.

\section{Methodology}

Six sessions of therapeutic plasma exchange (TPE) were done consecutively on our index patient using cobe spectra version 6.1 by continuous centrifugation technique using two venous lines (access and return lines). The femoral vein was utilised with the aid of a double lumen dialysis catheter. Anticoagulation with citrate (ACD) was systematically used. The replacement fluid employed in the TPE procedures was 5\% albumin for the first 3 sessions with an average replacement volume of 3.5 litres over one hour as calculated by the Cobe spectra machine. The subsequent three sessions were carried out using group compatible fresh frozen plasma which had been screened and found negative for transfusion transmissible infections (TTIs). The replacement volume of FFP was about 3.5 litres replaced over a longer duration of three hours as estimated by the Cobe spectra.

Being ventilator bound due to severe respiratory distress, the patient was co-managed in the apheresis unit of our hospital on ventilatory support by the Anaesthesia, Neurology and Haematology. A careful monitoring of hemodynamic parameters was done and signs of any complications during or following TPE were looked out for so as to institute urgent interventions. Calcium replacement with $1000 \mathrm{mg}$ of oral calcium (CAC 1000) was given to the patient at the commencement of the procedure to avoid citrate toxicity from use of FFP. The patient's haemogram, serum electrolytes, total protein, and albumin were monitored before and after each procedure. The procedure was well tolerated during all the six sessions.

\section{Case Summary}

The patient is a 47year old man, who was diagnosed with MG following a history of progressive ptosis, about 20 years prior to presentation to our health facility. He was admitted by the neurology team into the ICU for mechanical ventilator support on account of severe myasthenia crisis (class V). His chief complaints were severe difficulty with breathing and swallowing. Examination at presentation revealed a conscious but lethargic middle aged man in severe respiratory distress, bilateral ptosis with mild pallor. He was immediately commenced on mechanical ventilatory support with endotracheal intubation and later had tracheostomy done. He was dysphonic and also had muscle poor power in both upper and lower limbs. The clinical impression at this stage was severe myasthenia crisis likely complicated by sepsis with the focus being upper respiratory tract.

Haematology was invited for therapeutic plasmapheresis, since available management options offered could not effectively restore normal respiration. The options explored included use of IV Neostigmine, physiotherapy, salbutamol Nebulization, mechanical ventilation and antibiotic therapy. Hence, he became ventilator bound. TPE kit was sourced for by the patient's relation from another tertiary institution.

The patient had the first TPE after one month into his admission with significant improvement in clinical condition within one week of the $1^{\text {st }}$ session of plasmapheresis. He was able to sit out of bed, eat with very minimal dysphagia and was off ventilator support except for occasional nebulization. This improvement could not be sustained beyond four days because the apheresis machine developed a fault. The patient then deteriorated with 
severe dyspnoea, dysphagia and dysphonia; necessitating recommencement of mechanical ventilation, neostigmine and pyridostigmine.

It took about 6 weeks before the apheresis machine could be fixed. Thereafter, the patient had five more sessions of TPE. The improvement after these sessions was minimal, the patient became dependent on mechanical ventilatory support.

Intravenous immunoglobulin was used after the six sessions of TPE, after which he gradually improved with full recovery three weeks post infusion of IV Immunoglobulin. He was discharged home after 22 weeks on admission. He is now fully ambulant and back to normal activities.

\section{Discussion}

Myasthenia gravis is a rare autoimmune disorder said to be commoner in females in cases diagnosed below age 50 years and reverse in older age group. Our index patient is a rare case of a male diagnosed with MG at younger age of 26 years. He had had a mild course of the disease until about age 42 years when he started having occasional exacerbation of the MG. This necessitated consideration of thymectomy which was done overseas in conjunction with several sessions of TPE about three years prior to present illness. Cases of MG crisis have been reported in some patients after thymectomy. Most of the reported cases developed the acute myasthenia crisis within 72 hours of thymectomy, although we do not know the post thymectomy details of this patient, he appeared to have had some form of remission for about 3 years before the index crisis.

Immunomodulatory therapy in MG are especially used when rapid improvement is required such as in cases of myasthenic crisis and perioperative optimization of strength prior to thymectomy. The immunomodulatory therapeutic options include TPE and use of IVIG. The efficiency of TPE in MG is due to removal of autoimmune antibodies to acetylcholine receptor, leading to short-term improvement of neuromuscular junction transmission, muscular strength and motor performance. However, a rapid reduction of the autoantibodies may sometimes lead to a rebound excessive production of same antibodies [11]. This may explain why this patient was unable to sustain the initial clinical improvement observed after the first session of TPE. Rebound production of antibodies is also thought to render the replicating pathogenic cells more vulnerable to immunosuppressive drugs [11]. For this reason, TPE is sometimes performed to enhance the effectiveness of immunosuppressive drugs. Our index patient showed remarkable and sustained recovery following infusion of IVIG given within a short interval after the $6^{\text {th }}$ TPE session.

Another possibility for non-sustainability of the initial response to TPE in this patient could be the irregularity of the administration of TPE. Therapeutic plasma exchange has been reported to be more effective when given in short interval especially on alternate day [12]. The irregularity of TPE in our patient was multifactorial, ranging from unavailability of Cobe spectra TPE kit, faulty cobe spectra machine with delay in repair due to lack of on-site trained engineers, lack of backup equipment to the financial constraint on the part of the patient.

\section{Conclusion}

TPE is an essential immunomodulatory therapy in the management of acute myasthenia crisis when given consecutively over short intervals. It could be given alone or in combination with immunosuppressive drugs like IVIG. Rebound overproduction of the offending autoantibodies could follow TPE. The use of immunosuppressive therapy to counter rebound antibody production should be considered.

\section{References}

[1] Boon, N. A., Colledge, N. R., and Walker, B. R., 2006. Davidson principles and practice of Medicine. 20th ed. Philadelphia, USA: Churchill Livingstone Elsevier.

[2] Conti-Fine, B. M., 2006. "Myasthenia gravis: Past, present and future." J Clin Invest., vol. 116, p. 2843.

[3] McGrogan, A., Sneddon, S., and de Vries, C. S., 2010. "The incidence of myasthenia gravis: A systematic literature review." Neuroepidemiology, vol. 34, pp. 171-183.

[4] Won, H. K., Jung, H. K., Eun, K. K., Sang, P. Y., Kyung, K. K., and Won, C. K., 2010. "Myasthenia gravis presenting as isolated respiratory failure: A case report." Korean J. Intern Med., vol. 25, pp. 101-104.

[5] Scneider-Gold, C., Krenzer, M., Klinker, E., Mansoyuri-Thalegani, B., Mullges, W., and Toyka, K. V., 2016. "Immunoadsorption versus plasma exchange versus combination for treatment myasthenic deterioration." Ther Adv Neurol Disord., vol. 9, pp. 297-303.

[6] Lockwood, C. M., Worledge, S., Nicholas, A., Cotton, C., and Peters, D. K., 1979. "Reversal ofimpaired Splenic function in patients with nephritis or vasculitis (or both) by plasma exchange." N. Engl. J. Med., vol. 300, pp. 524-530.

[7] Newson-Davis, J., Wilson, S. G., Vincent, A., and Ward, C. D., 1979. "Long term effects of repeated plasma exchange in Myasthenia Gravis." Lancet, vol. 1, pp. 464-468.

[8] Gajdos, P., Chevret, S., and Toyka, K. V., 2002. "Plasma Exchange for generalised myasthenia gravis." Available: $\quad$ https://www.cochrane.org/CD002275/NEUROMUSC_plasma-exchange-for-generalisedmyasthenia-gravis

[9] Abjah, U. M., Aken'ova, Y. A., and Ocheni, S., 2002. "Waldenstrom's macroglobulinaemia: modified plasmapheresis as treatment option." Nigerian Journal of Medicine, vol. 11, pp. 190-192.

[10] Talabi, O. A., Abjah, U. M., Ocheni, S., Akinyemi, O. A., Aken'ova, Y. A., and Ogunniyi, A., 2006. "Benefit of modified plasmapheresis in the management of myasthenia gravis: A case report." Niger $J$ Med., vol. 15, pp. 162-164. 
[11] Heatwole, C., Johnson, N., Holloway, R., and Noyes, k., 2011. "Plasma exchangeversus Intravenous Immunoglobulin for myasthenia crisis. An acute Hospital cost comparison study." Neuromuscul Dis, vol. 13, pp. 85-94.

[12] Carandina-Maffeis, R., Nucci, A., MarquesJr, J. F. C., Roveri, E. G., Pfeilsticker, B. H. M., and Garibaldi, S. G., 2004. "Plasmapheresis in the treatment of myasthenia gravis:retrospective study of 26 patients." Arq Neuro-Psiquiatr. Sao Paulo, vol. 62, pp. 391-395. 